\title{
Brief Report: Imitation of Meaningless Gestures in Individuals with Asperger Syndrome and High-functioning Autism
}

\author{
Heidi Stieglitz Ham • Martin Corley • \\ Gnanathusharan Rajendran · Jean Carletta · \\ Sara Swanson
}

\begin{abstract}
Nineteen people with Asperger syndrome (AS)/ High-Functioning Autism (HFA) (ages 7-15) were tested on imitation of two types of meaningless gesture: hand postures and finger positions. The individuals with AS/ HFA achieved lower scores in the imitation of both hand and finger positions relative to a matched neurotypical group. The between-group difference was primarily accounted for by performance on a test of visual motor integration, together with a hand imitation deficit which was specifically due to errors in body part orientation. Our findings implicate both visuomotor processes (Damasio and Maurer, 1978) and self-other mapping (Rogers and Pennington, 1991) in ASD imitation deficits. Following Goldenberg (1999), we propose that difficulties with body part orientation may underlie problems in meaningless gesture imitation.
\end{abstract}

Imitation deficits in individuals with an Autism Spectrum Disorder (ASD) have been well documented (see Williams et al. 2004; Rogers and Williams 2006, for reviews). Rogers and Pennington (1991) suggest that a primary deficit in imitation may affect the development of symbolic thinking, emotion-sharing, and joint attention, which in itself is a precursor of theory of mind (Charman 2003). Williams et al. (2001) have suggested that the faulty development of the mirror system in individuals with ASD could be responsible for the noted deficits in self-other mapping and hence the social cognitive deficits found in this population.

Imitation comes under the umbrella of interpersonal matching, and has been defined by Moody and McIntosh (2006) as the copying of the action of a model rather than matching the outcome of the action by different means (cp. emulation). Imitation in this sense is purposeful because a choice to imitate has been made (Nadel 2006). Further, imitation can be subdivided into the imitation of meaningful and meaningless gestures, which can be considered separately. The advantage of this differentiation for autism research is that production of meaningless gestures requires matching from one person to another. In contrast to the production of meaningful gestures, imitation of meaningless gestures cannot rely on prior knowledge or on the meaning of the gesture itself (Goldenberg and Karnath 2006). In fact, novel gestures have been proposed to be the most genuine test of imitation because representations cannot be elicited from long-term memory (Tomasello 1999; Goldenberg and Strauss 2002).

Here, we report an exploratory study based on tests of the imitation of meaningless gestures adapted from Goldenberg (1999). In studies of apraxic patients Goldenberg (1999; Goldenberg and Strauss 2002) found that right-brain damaged patients were relatively more impaired in imitating 
finger positions than hand postures, and that left-brain damaged patients demonstrated the opposite pattern (cp. Della Sala et al. 2006). Goldenberg suggested that hand and finger imitation may tap into different systems: Imitation of the hand may recruit knowledge of the human body and 'body part coding', taking into consideration differences between the imitatee and imitators' bodies, such as size, height, shape, and orientation (Goldenberg and Hermsdorfer 2002). In contrast, imitation of finger positions is hypothesised to rely primarily on detailed visuo-spatial analysis.

In the present study, we compare the performance of children with ASD to that of matched control participants on the imitation of meaningless hand and finger gestures. Although autism is a developmental, and not an acquired disorder, the potential distinction between imitative systems observed in the apraxia literature offers a starting point for investigating imitative problems. Observing how ASD participants perform may provide some insight into the specific bases of their imitation difficulties.

In addition to the hand and finger imitation tests, a number of other measures were taken. Our research questions were two-fold. First, do children with autism show impairments in imitation of meaningless gesture that are not explained through other perceptual or motor mechanisms? Second, if children with autism do fail to imitate meaningless gestures, then what systems might contribute to this failure?

\section{Method}

\section{Participants}

Nineteen children, ages 7-15, diagnosed with Asperger syndrome (AS) (16 of the 19) or as having High-Functioning Autism (HFA) (3), were recruited through the Autism Society of Southeastern Wisconsin and were tested in the Department of Neurology and Neurosciences at the Medical College of Wisconsin. All children met DSM-IV (American Psychiatric Association 1994) diagnostic criteria for autism and were diagnosed prior to referral to the study. No participants were known to have a diagnosis comorbid with any other disorder. Participants were tested using the ADOS Modules 3 or 4 as appropriate (Autism Diagnostic Observation Schedule: Lord et al. 1999), and a parent interview was conducted using the SCQ (Social Communication Questionnaire: Rutter et al. 2003). Participants' ADOS communication and social scores ranged from 12 to 26 (mean 17.1); SCQ scores ranged from 15 to 34 (mean 23.5).

Twenty-three typically developing children (TD), ages 7-15, were tested in order to match with the participants with AS/HFA. Subsequent testing showed no differences on matching criteria between the 23 TD and 19 ASD participants (PIQ: $\mathrm{F}(1,41)=2.29, p=.14$; CA, VIQ, FSIQ: Fs $<1$ ), and we therefore report analyses based on all 42 participants below. Table 1 provides details of the group characteristics.

Prior to the meaningless gesture testing, participants were evaluated using the Wechsler Abbreviated Scale of Intelligence (WASI: Wechsler 1999), the Beery Visual Motor Integration Test (VMI), and the Beery Visual Perceptual Subtest (VP: Beery and Beery 2004), and a specifically designed hand and finger perceptual matching task. The VMI and VP are paper and pencil tests that require participants to either copy or match geometric shapes of increasing complexity.

The imitation and perceptual matching tasks were based on photographic stills of hand and finger postures, after Goldenberg (1999). Each hand posture still showed the upper body, arm, hand, and face of a child actor producing a gesture. Similar to Goldenberg's stimuli, these stills differed only in the hands' positional relationships to the face and head, keeping the finger positions constant. The pictures of finger positions showed only the relevant hand and fingers. These varied only in the fingers' positions relative to each other.

In the matching tasks, participants viewed stills of hand postures and finger positions on a laptop. In each trial, the participants were shown one target photo (either hand posture or finger position) on the left of the screen, together with a still showing a matching gesture and three foils on the right. Consistent with Goldenberg (1999), each trial comprised photos of different people from different angles. Participants viewed ten hand and ten finger targets, and were asked to choose the matching still photo on each trial, resulting in a maximum ten correct matches for each of finger and hand matching.

Meaningless gesture imitation was assessed using two tasks: Imitation of hand postures and imitation of finger positions. These stimuli were also presented via laptop computer. The participants viewed ten hand posture stills and ten finger positions, which they were asked to imitate. Each participant was allowed two attempts to imitate each gesture; one with each hand. The participants were videotaped, and the recordings were subsequently coded by two raters, one of whom was blind to the experimental hypothesis and the other of whom was the experimenter (HSH). The posture achieved at the end of each attempted gesture was coded as correct or incorrect. For a posture to be considered correct, it had to conform to the following properties: the hand had to be in the same shape as the model (form) as well as in the same position in relation to the various body parts (body part orientation). The orientation had to be in the same plane and the response could not be rotated more than $180^{\circ}$ (rotation). All other gestures 
Table 1 Participants' characteristics

\begin{tabular}{|c|c|c|c|c|c|c|}
\hline \multirow{3}{*}{$\begin{array}{l}\text { Table } 1 \text { Participants' } \\
\text { characteristics }\end{array}$} & & & & & & \\
\hline & Participants $^{\mathrm{a}}$ & CA & VIQ & PIQ & FSIQ & Gender \\
\hline & \multicolumn{6}{|l|}{ ASD } \\
\hline & M & 12.1 & 106.0 & 102.5 & 106.0 & \multirow[t]{3}{*}{$17 \mathrm{M} / 2 \mathrm{~F}$} \\
\hline & SD & 2.35 & 19.0 & 22.7 & 21.0 & \\
\hline & Range & $7.6-15$ & $81-144$ & $72-155$ & $79-153$ & \\
\hline & \multicolumn{6}{|l|}{ Control } \\
\hline & M & 12.0 & 107.5 & 112.8 & 111.4 & \multirow[t]{3}{*}{$21 \mathrm{M} / 2 \mathrm{~F}$} \\
\hline & SD & 2.12 & 12.9 & 18.8 & 16.5 & \\
\hline $\begin{array}{c}n=19 \mathrm{ASD} ; 23 \mathrm{TD}= \\
\text { tynically develoning }\end{array}$ & Range & $7.3-15.8$ & $87-134$ & $69-143$ & $70-139$ & \\
\hline
\end{tabular}

a $n=19$ ASD; 23 TD = typically developing

were coded as "incorrect", and errors in each of the categories above (form, body part orientation, rotation) were noted. Seven examples of each error type were coded for inter-rater reliability, resulting in $80 \%$ agreement (rising to $100 \%$ when disagreements had been discussed).

\section{Results}

Because the preferred hand was used for the first production of each hand or finger imitation, we first conducted two mixed ANOVAs, with factors of attempt (first or second; within), and group (ASD or control; between). Since there were no effects involving attempt for either finger or hand [all Fs $<1$ ] our subsequent analyses were collapsed across this factor.

Other than the imitation tasks, we analyzed participants' performance on four other measures which had not been used in group matching (Table 2 summarises participants' scores). Participants did not differ on VP $(\mathrm{F}<1)$; however, ASD participants performed significantly worse on VMI $[\mathrm{F}(1,40)=15.328, p<.001]$, and a MANOVA established that they performed worse on hand and finger matching $[\mathrm{F}(1,39)=8.506, p<.001]$, with independent deficits in hand matching $[\mathrm{F}(1,40)=10.39, p=.003]$ and finger matching $[\mathrm{F}(1,40)=5.094, p=.03]$.

A between group MANOVA which used hand imitation and finger imitation scores as dependent variables showed that the AS/HFA participants performed significantly worse than controls overall $[\mathrm{F}(1,39)=9.639, p<001]$. This was due to independent deficits in hand imitation [6.8 vs. 9.0 correct for AS/HFA and TD participants respectively; $\mathrm{F}(1,40)=p<.001]$ and in finger imitation [7.5 vs. 8.9; $\mathrm{F}(1,40)=9.85, p=.003]$.

To establish which variables best accounted for group differences, we entered all six variables described above (VMI; VP; hand and finger matching and imitation) into a stepwise logistic regression predicting group membership. This yielded a 2-factor model which improved prediction by $26.2 \%$, to $81.0 \%$ correct, over the null model. Other than VMI, which improved prediction by $16.6 \%$, only hand imitation significantly improved the model [by an additional 9.6\%: for the two-factor model, Odds Ratio $=.898$, $p=.019$ for $\mathrm{VMI}$; Odds Ratio $=.488, p=.021$ for hand imitation] suggesting that these were the only two factors that could usefully distinguish participant groups. Hand imitation contributes independently to between-group differences; for each extra point scored for a correct hand imitation, participants are $51 \%$ less likely to belong to the ASD group. Since VMI measures visuomotor integration, we attribute the independent contribution of hand imitation to other aspects of the task.

To further investigate the deficit in hand imitation, we explored the reasons why the hand imitative gestures had been coded as incorrect. Based on Goldenberg and Hermsdorfer (2002) we hypothesised that body part orientation
Table 2 Participants' performance on Visual Motor Integration (VMI), Visual Perception (VP), and measures of hand and finger matching and imitation

a $n=19$ ASD; $23 \mathrm{TD}=$ typically developing

\begin{tabular}{lllllll}
\hline Participants $^{\mathrm{a}}$ & VMI & VP & Hand match & Finger match & Hand imitation & Finger imitation \\
\hline ASD & & & & & & \\
M & 87.6 & 25.8 & 6.0 & 6.5 & 6.8 & 7.4 \\
SD & 10.4 & 2.6 & 2.0 & 2.3 & 2.5 & 2.1 \\
Range & $72-109$ & $20-30$ & $2-10$ & $4-10$ & $1.5-10$ & $3.5-10$ \\
Control & & & & & & \\
M & 102.4 & 26.5 & 8.0 & 7.8 & 9.0 & 9.0 \\
SD & 13.5 & 2.5 & 1.5 & 1.3 & 0.9 & 1.0 \\
Range & $78-136$ & $19-30$ & $4-10$ & $5-10$ & $7-10$ & $7-10$ \\
\hline
\end{tabular}


errors, but not form or rotation errors, would predict group membership. We performed a stepwise logistic regression predicting group from these three classes of error. The analysis established that the inclusion of body part orientation errors improved prediction accuracy over the null model by $23.8 \%$, to $78.6 \%$, with no other variables making a significant contribution [Odds Ratio $=2.91, p=.004$ ]

\section{Discussion}

As a group, the participants with AS/HFA achieved lower scores in the imitation of both hand postures and finger positions, as well as demonstrating poorer performance in the visuo-motor integration (VMI) and hand and finger matching tasks. Additionally, a closer inspection of the evidence established that the difference between groups was largely accounted for by performance in tests of VMI and hand imitation. The finding that VMI accounted for the largest increase in prediction accuracy points to differences in visuomotor processing (e.g., Damasio and Maurer 1978). There are, however, aspects of hand imitation that account for group membership independently of VMI. Analyses of the different types of errors observed in hand imitation suggest that body part orientation errors are in fact the major contributing predictor of betweengroup differences.

Although the patterns of performance demonstrated by the group with AS/HFA differ from those reported for adult neurological patients (Goldenberg 1999), it is difficult to draw comparisons with this population, given the different natures of the disorders. Nevertheless, body part orientation errors in the imitation of meaningless gestures implicate a specific system underlying a general imitative deficit in autism such as that hypothesised by Rogers and Pennington (1991). Rogers and Pennington suggest that individuals with autism have difficulty "seeing others as a template of the self". Our findings suggest that this difficulty may be, in part, due to problems in determining the relations of body parts to each other (cf. Goldenberg and Hermsdorfer 2002). In this respect, it is important that the gestures imitated in the present study are meaningless. Where gestures have meaning, their imitation may rely on other processes (for example, meaningful gestures may implicate a gestural 'lexicon' in addition to the more basic processes investigated here). Similarly, where imitation is part of a social communicative act, there may be other factors underlying any difficulty (such as interpersonal connectedness: Hobson and Lee 1999). When social and lexical factors are removed, ASD participants still have problems in imitating the postures adopted by others.

A further question remains of whether visuospatial processes, separately identified as leading to imitative deficits in Goldenberg's sample of apraxic patients, may also be implicated in some individuals with ASD. In this context, we note that our AS/HFA participants performed worse than controls on finger imitation tasks, although finger performance did not reliably predict group membership. Taken together, our findings suggest that future investigations of imitative deficits in ASD could usefully take into account Goldenberg's (1999) theory that imitation in its purest form is 'body part specific'.

\section{References}

American Psychiatric Association. (1994). Diagnostic and statistical manual of mental disorders (4th ed.). Washington, DC.

Beery, K., \& Beery, N. (2004). The Developmental Test of Visual Motor Integration. Manual Los Angeles, CA: Western Psychological Services.

Charman, T. (2003). Why is joint attention a pivotal skill in autism? Philosophical Transactions of the Royal Society B: Biological Sciences, 358(1430), 315-324.

Damasio, A. R., \& Maurer, R. G. (1978). A neurological model for childhood autism. Archives of Neurology, 35, 777-786.

Della Sala, S., Faglioni, P., Motto, C., \& Spinnler, H (2006). Hemisphere asymmetry for imitation of hand and finger movements: Goldenberg's hypothesis reworked. Neuropsychologia, 44, 1496-1500.

Goldenberg G. (1999). Matching and imitation of hand and finger postures in patients with damage in the right or left hemispheres. Neuropsychologia, 37(5), 559-566.

Goldenberg, G., \& Hermsdorfer, J. (2002). Imitation, apraxia, and hemisphere dominance. In A. Meltzoff \& W. Prinz (Eds.), The imitative mind (pp. 331-347). Cambridge, UK: Cambridge University Press.

Goldenberg, G., \& Strauss S. (2002). Hemisphere asymmetries for imitation of novel gestures. Neurology, 59, 893-897.

Goldenberg, G., \& Karnath, H.-O. (2006). The neural basis of imitation is body specific. Journal of Neuroscience, 26, 6282-6287.

Hobson, R. P., \& Lee, J. (1999) Imitation and identification in autism. Journal of Child Psychology and Psychiatry, 40, 649-659.

Lord, C., Rutter, M., DiLavore, D., \& Risi, S. (1999). Autism Diagnostic Observation Schedule (ADOS). Los Angeles, Calif: Western Psychological Services.

Moody, E., \& McIntosh, D. (2006). Imitation in autism findings, controversies. In S. Rogers \& J. H. Williams (Eds.), Imitation and the social mind (pp. 71-88). New York, NY: Guilford Press.

Nadel, J. (2006). Does imitation matter to children with autism? In S. Rogers \& J. H. Williams (Eds.), Imitation and the social mind (pp. 118-134). New York, NY: Guilford Press.

Rogers, S. J., \& Pennington, B. F. (1991). A theoretical approach to the deficits in infantile autism. Development and Psychopathology, 3, 137-162.

Rogers, S., \& Williams, J. H. (2006). Imitation in autism findings, controversies. In S. Rogers \& J. H. Williams (Eds.), Imitation and the social mind (pp. 277-303). New York, NY: Guilford Press.

Rutter M., Bailey A., \& Lord, C. (2003). SCQ: The social communication questionnaire manual Los Angeles, CA: Western Psychological Services.

Tomasello, M. (1999). The cultural origins of human cognition. Cambridge, MA: Harvard University Press. 
Wechsler, D. (1999). Wechsler abbreviated scale of intelligence (WASI). San Antonio, TX: The Psychological Corporation.

Williams, J., Whiten, A., \& Singh, T. (2004). A systematic review of action imitation in autistic spectrum disorder. Journal of Autism and Developmental Disorders, 34, 285-299.
Williams, J., Whiten, A., Suddendorf, T., \& Perrett, D. (2001). Imitation, mirror neurons, and autism. Neuroscience and Biobehavioral Reviews, 25, 577-596. 